\title{
MEMORY IMPROVEMENT WITH TREATMENT OF HYPOTHYROIDISM
}

\section{KAREN J. MILLER}

Department of Psychiatry and Biobehavioral Sciences UCLA NPI-H Los Angeles, California, USA

\section{THOMAS D. PARSONS}

Department of Psychiatry and Biobehavioral Sciences UCLA NPI-H Los Angeles, California, USA

and

Department of Neurology School of Medicine University of North Carolina at Chapel Hill Chapel Hill, North Carolina, USA

\section{PETER C. WHYBROW}

Department of Psychiatry and Biobehavioral Sciences UCLA NPI-H

Los Angeles, California, USA Advisory Council (TRAK) [grant SYN-0400-13] (Dr. Bauer).

Address correspondence to Thomas D. Parsons, Ph.D., Department of Neurology, CB \# 7025 , University of North Carolina School of Medicine, 3114 Bioinformatics Building Chapel Hill, NC27599-7025, USA. E-mail: tparsons@neurology.unc.edu 
KATJA VAN HERLE

NATALIE RASGON

Department of Medicine

UCLA Division of Endocrinology

Los Angeles, California, USA

and

Department of Psychiatry and Behavioral Sciences

Stanford School of Medicine

Palo Alto, California, USA

ANDRE VAN HERLE

DOROTHY MARTINEZ

Department of Medicine

UCLA Division of Endocrinology

Los Angeles, California, USA

DAN H. SILVERMAN

Department of Molecular and Medical Pharmacology

Los Angeles, California, USA

\section{MICHAEL BAUER}

Department of Psychiatry and Biobehavioral Sciences UCLA NPI-H

Los Angeles, California, USA

and

David Geffen School of Medicine

UCLA

Los Angeles, California, USA

and

Department of Psychiatry and Psychotherapy

Charité-University Medicine Berlin

Campus Charité Mitte

Berlin, Germany 
The consequences of inadequate thyroid hormone availability to the brain and treatment effects of levothyroxine on cognitive function are still poorly understood. This study prospectively assessed the effects of thyroid replacement therapy on cognitive function in patients suffering from biochemical evidenced, untreated hypothyroidism. Significant effects between the untreated hypothyroid group and control group were limited to verbal memory retrieval. When assessing the effects of 3-month treatment, results revealed that the treated hypothyroid group had significant increased verbal memory retrieval. Results suggest that specific memory retrieval deficits associated with hypothyroidism can resolve after replacement therapy with levothyroxine.

Keywords cognition, hypothyroidism, levothyroxine, memory, neuropsychological tests

Although hypothyroidism is often associated with mood disturbances and cognitive impairment, several researchers have suggested the reversibility of these cognitive and mood disturbances with thyroid replacement therapy (Crown, 1949; Whybrow et al., 1969; Haggerty et al., 1990; Osterwil et al., 1992; Whybrow \& Bauer, 2005; Bunevicius \& Prange, 2000; Burmeister et al., 2001; Bjerke et al., 2001; Smith et al., 2002).

Memory impairment and improvement with thyroid treatment have been examined repeatedly in the past. Osterwil et al. (1992) reported, in a study of 29 participants, that there was a correlation between hypothyroidism and a significant impairment in verbal associative learning and retention, which improved with treatment. Monzani et al. (1993) found with 14 participants that their contextual verbal memory significantly improved with thyroid replacement, while depression levels declined (although not statistically significant). Jaeschke et al. (1996) reported that there was a significant improvement in verbal memory for their 18 participants, using a memory composite of contextual and associative memory tasks; processing speed did not improve with levothyroxine treatment. Baldini et al. (1997) found that their 19 participants had impaired memory during the hypothyroid state, but were not impaired for processing speed and depression; this memory deficit significantly improved with treatment. Bunevicius and Prange (2000) treated 26 hypothyroid patients and reported improvement in mood and cognition (tasks of working memory and visual scanning). Burmeister et al. (2001) found that discontinuing thyroid hormone treatment for 13 thyroid cancer patients was associated with impairment for specific verbal memory retrieval deficits as seen on a rote list learning task, as compared to no significant memory deficits when successfully treated. Bjerke et al. (2001) also found 
that impaired mood and short-term memory in four cases of hypothyroidism improved with treatment, but the patients did not reach premorbid levels of functioning.

In contrast to those who report improvement with treatment, Mennemeier et al. (1993) and Leentjens and Kappers (1995) reported that in specific case studies the baseline memory functions remained impaired even with treatment when compared to normals. Furthermore, several researchers also reported that dementia associated with severe hypothyroidism may not always be reversible (Dugbartery, 1998; Whybrow \& Bauer, 2005).

The current study explored whether specific memory retrieval deficits would be exhibited by the hypothyroid subjects compared to the controls and if these memory deficits would reverse with thyroid replacement treatment. Specifically, it was proposed that if memory deficits were associated with hypothyroidism in this study, they would improve to "normal levels" (average range), although the controls would not demonstrate a significant change in their performance as they already would be exhibiting normal memory performances.

\section{MATERIAL AND METHODS}

\section{Study Design}

This study was approved by the UCLA institutional review board. It prospectively tested the effects of thyroid replacement treatment with levothyroxine $\left(\mathrm{L}-\mathrm{T}_{4}\right)$ in patients with untreated hypothyroidism on cognitive function. Two groups (hypothyroid patients and healthy controls) were tested with measures of mood and cognitive functioning at two time points. For the hypothyroid group, the times were before and after the course of 3-month $\mathrm{L}-\mathrm{T}_{4}$ treatment. The control group was tested at corresponding times to provide a measure of order effects, but was not treated with thyroid hormone.

\section{Subjects}

The research participants were recruited over one year, through flyers that described the study. Flyers were given to patients with hypothyroidism, admitted to the UCLA Division for Endocrinology Clinics, and also were posted at the UCLA Medical Center. All consecutively admitted patients and controls were screened for and, if appropriate, offered participation in the study. Screening procedures included a complete medical and physical examination, 
a routine laboratory evaluation (blood count, blood chemistry, urine drug toxicology), magnetic resonance imaging (MRI), and a comprehensive neuropsychiatric evaluation. Neuropsychiatric assessment comprised a psychiatric interview supplemented by a structured interview for DSM-IV diagnosis, the M.I.N.I. International Neuropsychiatric Interview, Version 5.0 (Sheehan, 1998). As part of this project a standard neuropsychological battery was administered.

Hypothyroid patients with impaired cognition of both sexes who were invited to continue into the treatment phase of the study were between 18 and 55 years old. Further inclusion criteria were as follows: Hypothyroid status was confirmed by elevated basal TSH levels. Participants must have been considered by the investigator to be compliant with investigations and appointments, must have had an educational level and a degree of understanding such that they could meaningfully communicate with the investigator and ensured that they understand the study. All participants were required to have given written informed consent.

The exclusion criteria were: endocrine or autoimmune diseases other than hypothyroidism, myxedema coma, pregnant or lactating women who had given birth in the past 12 months or less (to exclude antithyroid antibodies positive postpartum thyroiditis), women of childbearing potential not using contraception, psychotic features, a history of bipolar disorder or schizoaffective disorder, intake of thyroid hormones or corticosteroids in previous two months, participants judged clinically to be a serious suicide risk, and participants who were not facile in English.

Healthy control subjects were recruited through flyers at the UCLA Medical Center and advertisements in the campus newspaper. They were in good general health and underwent the same psychiatric screening procedures as described earlier for hypothyroid subjects. The inclusion criteria were: (1) age between 18 and 55 years, (2) no current or past mental disorder on the basis of the M.I.N.I. Neuropsychiatric Interview and a negative history of major organic brain disease, (3) score of 3 or less on the $\mathrm{HRSD}_{21}$ throughout the study, (4) no intake of any hormonal agents or psychotropic agents for at least 4 months prior to study, and (5) no history of endocrine, cardiac, or other severe medical disease.

In both groups, current dependence on an addictive substance or remarkable substance abuse with a positive urine test were also exclusionary. Fourteen subjects with hypothyroidism and ten age-matched healthy control subjects gave written informed consent and participated in this study. Participants were paid for participation. 


\section{Procedures}

Before initiation of thyroid replacement treatment, participants were administered a comprehensive neuropsychological test battery and clinician-rated mood evaluation (see later). Thyroid replacement therapy and titration of TSH was performed routinely by experienced endocrinologists at the UCLA Division of Endocrinolgy (K.V.H., A.V.H., D.M.). The second neuropsychiatric assessment was performed approximately three months after initiation of levothyroxine treatment and after patients had achieved euthyroid status as evidenced by routine laboratory measurement of thyroid status.

Neuropsychological Test Battery and Mood Ratings. The neuropsychological battery included a diverse collection of instruments. Verbal memory was assessed with the Logical Memory test from the Wechsler Memory Scale_-III (WMS_III; Wechsler, 1987) and the California Verbal Learning Test (CVLT; Delis et al., 2000). Nonverbal memory was assessed with the Rey-Osterrieth Complex Figure test (Osterrieth, 1944). Attention was assessed with the Trail-Making Test A (Army Individual Test Battery, 1944). The Trail-Making Test B (Army Individual Test Battery, 1944) and the Controlled Oral Word Association (COWA; Benton \& Hamsher, 1989) evaluated executive functioning. Language skills were tested with Animal Naming (category or semantic verbal fluency; Goodglass \& Kaplan, 1983). These tests are all commonly used for neuropsychological assessment of these cognitive processes and as such have widely used normative information available.

The clinician-rated Hamilton Rating scale for Depression (HAM-D; Hamilton, 1967) was utilized to assess mood and psychological functioning across occasions. The HAM-D is an established psychological symptom inventory that provides an indication of depression and, over time, provides a valuable guide to progress. A total index of symptoms can be created from the HAM-D, and is perceived as an indication of symptom breadth in reflecting the total number of items endorsed.

Statistical Analysis. Given the similarity of participants in both the hypothyroidism group and the control group on age, sex, and education (see Table 1), no correction for these variables was employed. To assess which patterns of performance were retained for the hypothyroidism group and the control group participants, the data analysis was completed in three stages. In the first stage, the reference distribution is performance of each group (hypothyroidism and control) before the administration of levothyroxine. $T$-tests for independent 
Table 1. Demographic characteristics of hypothyroid subjects and controls

\begin{tabular}{lcc}
\hline & $\begin{array}{c}\text { Hypothyroid } \\
\text { subjects } n=14\end{array}$ & $\begin{array}{c}\text { Healthy controls } \\
n=10\end{array}$ \\
\hline Age (years) & & \\
Gender (Female:Male) & $42.8 \pm 12.4$ & $41.0 \pm 12.0$ \\
Subject's education (years) & $11: 3$ & $8: 2$ \\
Type of education & $15.8 \pm 2.7$ & $16.3 \pm 1.5$ \\
$\quad$ Postgraduate & & \\
Completed college & 4 & 3 \\
Some college & 3 & 3 \\
$\quad$ High school graduate & 4 & 0 \\
Race & 2 & $5 / 10$ \\
Caucasian (non-Hispanic) & & $1 / 10$ \\
Caucasian of South American descent & $12 / 14$ & $2 / 10$ \\
African American & $1 / 14$ & $2 / 10$ \\
Asian & $1 / 14$ & 9 \\
Handedness & $0 / 14$ & 0 \\
Right & & 1 \\
Left & 13 & $89 \pm 18$ \\
Ambidextrous & 1 & $1.4 \pm 0.5$ \\
Days between Time 1 and Time 2 test periods ${ }^{1}$ & 0 & $(1.1-2.4)$ \\
TSH (mcIU/ml) ${ }^{2}$ & $110 \pm 38$ & \\
(Range) & $31.5 \pm 56.2$ & \\
\hline
\end{tabular}

\footnotetext{
${ }^{1}$ Data shown are means $\pm \mathrm{SD}$.
}

$\mathbf{2}_{\text {[Reference range: } 4.9-1.4] \text {. }}$

samples were used to evaluate the significance of group differences on measures of cognition. In the second stage, repeated measures ANOVAs were used to test scores from two testing (Time 1 and Time 2) occasions for significant change. In stage three, group differences found to be significant were placed into hierarchical multiple regressions to control for the potentially confounding effects of mood, by forcing the HAM-D Scale total score into the equation prior to analyzing the effects of group. Two-tailed significance tests were used throughout.

\section{RESULTS}

The two groups did not differ significantly in age, sex, education, ethnicity, and handedness (Table 1). All the patients who received the L-T 4 treatment completed the study, and exhibited declines in depression scores on the 
clinician-rated HAM-D scale (17 items) $(12.8 \pm 8.1$ vs. $4.2 \pm 3.9 ; p<.05)$. At the time of the second cognitive assessment (Time 2), the mean $\mathrm{L}_{-} \mathrm{T}_{4}$ dose was $95.1 \pm 24.6 \mathrm{mcg} / \mathrm{day}$ and the TSH levels had decreased significantly with treatment (from $31.5 \pm 56.2 \mathrm{mcIU} / \mathrm{ml}$ to $5.2 \pm 9.2 \mathrm{mcIU} / \mathrm{ml} ; p<.01$ ).

Descriptive statistics for Time 1 and Time 2 of healthy controls and hypothyroid group on all neuropsychological tests are shown in Table 2. Repeated measures ANOVAs comparing healthy controls to hypothyroidism group on all neuropsychological tests are presented in Table 3. Results from stage one analysis revealed that although verbal group effects were found between the hypothyroidism and control groups, they were limited to verbal memory retrieval on the California Verbal Learning Test (CVLT). On Short Delay Free Recall $(p=.01)$, Long Delay Free Recall $(p=<.05)$ and Long Delay Cued Recall $(p=.01)$, significant differences remained between groups despite the limited statistical power of this study. Contrariwise, there were no significant results found between hypothyroidism and control groups on attentional or nonverbal verbal tasks.

Table 2. Descriptive statistics for Time 1 and Time 2 of healthy controls and hypothyroidism group on all neuropsychological tests*

\begin{tabular}{|c|c|c|c|c|c|c|c|c|}
\hline \multirow{3}{*}{$\begin{array}{l}\text { Test } \\
\text { CVLT Trial } 1\end{array}$} & \multicolumn{4}{|c|}{ Time 1} & \multicolumn{4}{|c|}{ Time 2} \\
\hline & \multicolumn{2}{|c|}{$\begin{array}{c}\text { Healthy } \\
\text { controls Mean } \\
\text { (SD) }\end{array}$} & \multicolumn{2}{|c|}{$\begin{array}{l}\text { Hypothyroid } \\
\text { group Mean } \\
\text { (SD) }\end{array}$} & \multicolumn{2}{|c|}{$\begin{array}{c}\text { Healthy } \\
\text { controls Mean } \\
\text { (SD) }\end{array}$} & \multicolumn{2}{|c|}{$\begin{array}{c}\text { Hypothyroid } \\
\text { group Mean } \\
\text { (SD) }\end{array}$} \\
\hline & 10.40 & 2.01 & 8.64 & 2.50 & 8.80 & 1.81 & 8.0 & 2.3 \\
\hline CVLT Trial 5 & 15 & 1.05 & 14.07 & 2.23 & 15.00 & 1.25 & 13.8 & 2.1 \\
\hline CVLT Total & 66.9 & 7.39 & 60.93 & 9.34 & 63.20 & 6.05 & 59.6 & 8.6 \\
\hline CVLT List B & 7.1 & 2.42 & 8.07 & 2.30 & 8.20 & 1.32 & 7.5 & 2.4 \\
\hline CVLT SD Free & 13.4 & 2.59 & 13.71 & 2.23 & 13.20 & 2.90 & 11.4 & 3.2 \\
\hline CVLT SD Cued & 14.8 & 1.32 & 14.43 & 1.65 & 14.50 & 1.78 & 12.8 & 2.1 \\
\hline CVLT LD Free & 14.9 & 1.29 & 14.14 & 1.51 & 14.40 & 1.90 & 12.6 & 2.2 \\
\hline CVLT LD Cued & 15 & 1.15 & 14.36 & 1.55 & 14.90 & 1.66 & 12.7 & 2.3 \\
\hline Logical Memory I & 31.1 & 6.42 & 29.07 & 6.94 & 28.90 & 8.41 & 26.0 & 5.5 \\
\hline Logical Mem. II & 27.9 & 6.62 & 27.07 & 7.19 & 26.60 & 7.97 & 22.4 & 7.0 \\
\hline Rey Copy & 31.3 & 2.45 & 32.79 & 3.91 & 31.10 & 5.43 & 33.4 & 3.0 \\
\hline Rey Delay & 20.55 & 7.14 & 18.71 & 7.59 & 18.70 & 6.32 & 18.8 & 5.0 \\
\hline FAS & 44.7 & 12.43 & 52.50 & 20.76 & 42.20 & 9.37 & 45.4 & 17.2 \\
\hline Animal naming & 21.6 & 4.77 & 18.93 & 6.99 & 22.60 & 5.08 & 19.9 & 6.4 \\
\hline Trails A & 24.5 & 5.58 & 26.21 & 6.99 & 30.50 & 7.76 & 29.9 & 7.0 \\
\hline Trails B & 52.7 & 17.38 & 60.43 & 26.33 & 80.90 & 34.02 & 65.5 & 21.2 \\
\hline
\end{tabular}

${ }^{*}$ For all analyses hypothyroidism group $n=14$; and control group $n=10$. 
Table 3. Repeated measures ANOVAs comparing healthy controls to hypothyroidism group on all neuropsychological tests*

\begin{tabular}{|c|c|c|c|c|c|c|}
\hline \multirow{3}{*}{$\begin{array}{l}\text { Test } \\
\text { Logical Memory } 1\end{array}$} & \multirow{2}{*}{\multicolumn{2}{|c|}{ Hypothyroid Time 1}} & \multirow{2}{*}{\multicolumn{2}{|c|}{ Hypothyroid Time 2}} & \multicolumn{2}{|c|}{ Interaction } \\
\hline & & & & & \multirow{2}{*}{$\frac{f}{0.23}$} & \multirow{2}{*}{$\begin{array}{c}p \\
\mathrm{~ns}\end{array}$} \\
\hline & 26.00 & $(5.46)$ & 29.92 & (6.67) & & \\
\hline Logical Memory 2 & 22.36 & $(7.04)$ & 27.42 & $(6.83)$ & 0.49 & ns \\
\hline Trails A & 29.90 & (7.01) & 25.50 & $(6.37)$ & 0.31 & ns \\
\hline Trails B & 65.50 & (21.19) & 57.21 & $(22.92)$ & 0.01 & ns \\
\hline Animal naming & 19.90 & $(6.40)$ & 20.04 & $(6.19)$ & 0.16 & ns \\
\hline FAS & 45.40 & $(17.23)$ & 49.25 & $(17.87)$ & 0.52 & ns \\
\hline CVLT Trial 1 & 8.00 & $(2.25)$ & 9.38 & $(2.43)$ & 0.38 & ns \\
\hline CVLT Trial 5 & 13.80 & $(2.08)$ & 14.46 & $(1.86)$ & 0.55 & ns \\
\hline CVLT Total & 59.60 & $(8.60)$ & 63.42 & $(8.92)$ & 2.43 & ns \\
\hline CVLT List B & 7.50 & $(2.38)$ & 7.67 & $(2.35)$ & 2.59 & ns \\
\hline CVLT SD Free & 11.40 & (3.18) & 13.58 & $(2.34)$ & 6.53 & .01 \\
\hline CVLT SD Cued & 12.80 & $(2.12)$ & 14.58 & $(1.50)$ & 1.47 & ns \\
\hline CVLT LD Free & 12.60 & $(2.17)$ & 14.46 & $(1.44)$ & 1.60 & $>.05$ \\
\hline CVLT LD Cued & 12.70 & $(2.30)$ & 14.63 & $(1.41)$ & 9.81 & .01 \\
\hline Rey Copy & 33.36 & $(2.98)$ & 32.17 & $(3.40)$ & 0.40 & ns \\
\hline Rey Delay & 18.75 & $(4.97)$ & 19.48 & $(7.31)$ & 0.78 & ns \\
\hline
\end{tabular}

${ }^{*}$ For all analyses hypothyroidism group $n=14$; and control group $n=10$.

Results from the second stage analysis revealed that hypothyroid treatment effects increased performance on the CVLT's Short Delay Free Recall $(p=.01)$, Long Delay Free Recall ( $p=<.05)$, and Long Delay Cued Recall $(p=.01)$. Significant differences remained between groups despite the limited statistical power of this study. Contrariwise, there were no significant results found between hypothyroidism and control groups on attentional or nonverbal verbal tasks. No practice effects were found. There were no significant differences between the control group and the treated hypothyroid group at time two of testing. In stage three of the analysis, after controlling for mood, as described in the section on data analytic strategy, significant differences remained between groups on CVLT's Short Delay Free Recall $(p=.02)$, and Long Delay Cued Recall ( $p=.002$ ), despite the limited statistical power of this study. The subtest on which no significant difference remained between groups was CVLT Long Delay Free Recall $(p=.15)$.

\section{DISCUSSION}

The untreated hypothyroid participants demonstrated a specific deficit in memory retrieval, with more difficulty retrieving verbal information after both 
a short and long delay, even after category cues were provided. The hypothyroid participants did not differ in terms of encoding new verbal information or the delayed recognition of this same information. The contrast between impairment in retrieval compared to intact encoding and recognition suggests that hypothyroidism may not impair cognition or memory globally, but has a distinct profile of memory retrieval deficits, which may implicate the prefrontal cortex. There were no other executive deficits, which are typically associated with depression or normal aging, such as sustained attention, set-shifting, response inhibition, and cognitive flexibility (Salthouse, 1991; Boone et al., 1995; Christiansen et al., 1997; Austin et al., 1999; Nebes, et al. 2000; Comijs et al., 2001).

The specific memory retrieval deficits resolved with the administration of levothyroxine, including improvement for both short and long delayed recall, and mood. Significant improvement was not found in the control group, ruling out practice effects. After treatment, there were no significant differences between the hypothyroid and control groups. Furthermore, when the results were controlled for depressive symptoms, there was still significant improvement in short and long delayed recall for the treated hypothyroid group, suggesting that improvement in memory was not directly linked to the treatment of depressive symptoms. These results suggest that although hypothyroidism can result in mood and cognitive deficits, treatment is critical and may resolve specific deficits. A recent research study in animals (rats) has suggested that there is an augmentative effect of thyroxine upon cognitive function, including enhanced memory and increased cholinergic activity in the frontal cortex and hippocampus (Smith et al., 2002). Recent advances in understanding the specific deficits associated with hypothyroidism, along with necessary treatment regimens, are critical for early detection and successful treatment, particularly given the potential of residual cognitive deficits or dementia with severe hypothyroidism.

Although this study extends the current understanding of the specific memory deficits associated with hypothyroidism and the improvement associated with thyroid hormone treatment, it is a small study that focused on short-term treatment. Therefore, large studies with additional follow-up measures are needed to confirm results.

\section{REFERENCES}

Army Individual Test Battery. (1944). Manual of directions and scoring. Washington, DC: War Department, Adjutant General's Office. 
Austin, M. P., Mitchell, P., Wilhelm, K., Parker, G., Hickie, I., Brodaty, H., Chan, J., Eyers, K., Milic, M., \& Hadzi-Pavlovic, D. (1999). Cognitive function in depression: A distinct pattern of frontal impairment in melancholia? Psychological Medicine, 29, 73-85.

Baldini, I., Vita, A., Mauri, M., Amodei, V., Carrisi, M., Bravin, S., \& Cantalamessa, L. (1997). Psychopathological and cognitive features of subclinical hypothyroidism. Prog. Neuropsychopharmacol. Biological Psychiatry, 21, 925-935.

Benton, A. L., \& Hamsher, K. (1994). Multilingual Aphasia Examination. Iowa City: AJA Associates.

Bjerke, S. N., Bjoro, T., \& Heyerdahl, S. (2001). Psychiatric and cognitive aspects of hypothyroidism. Tidsskr Nor Laegeforn, 121(20), 2373-2376.

Boone, K. B., Lesser, I. M., Miller, B. L., Wohl, M., Berman, N., Lee, A., Palmer, B., \& Back, C. (1995). Cognitive functioning in older depressed outpatients: Relationship of presence and severity of depression to neuropsychological test scores. Neuropsychology, 9(3), 390-398.

Bunevicius, R., \& Prange, A. J. (2000). Mental improvement after replacement therapy with thyroxine plus triiodothyroine: Relationship to cause of hypothyroidism. International Journal of Neuropsychopharmacology, 3, 167-174.

Burmeister, L. A., Ganguli, M., Dodge, H. H., Toczek, T., DeKosky, S. T., \& Nebese, R. D. (2001). Hypothyroidism and cognition: Preliminary evidence for a specific defect in memory. Thyroid, 11, 1177-1185.

Christensen, H., Griffiths, K., MacKinnon, A., \& Jacomb, P. (1997). A quantitative review of cognitive deficits in depression and Alzheimer-type dementia. Journal of the International Neuropsychological Society, 3, 631-651.

Comijs, H. C., Jonker, C., Beekman, A. T. F., \& Deeg, D. J. H. (2001). The association between depressive symptoms and cognitive decline in community-dwelling elderly persons. International Journal of Geriatric Psychiatry, 16, 361-367.

Crown, S. (1949). Notes on an experimental study of intellectual deterioration. British Medical Journal, 684-685.

Delis, D. C., Kramer, J. H., Kaplan, E., \& Ober, B. (2000). California Verbal Learning Test-second edition. New York: Psychological Corporation.

Dugbartey, A. T. (1998). Neurocognitive aspects of hypothyroidism. Archives of Internal Medicine, 158, 1413-1418.

Goodglass, H., \& Kaplan, E. (1983). The Assessment of Aphasia and Related Disorders ed. 2. Philadelphia: Lea \& Febiger.

Hagerty, J., Garbutt, J., Evans, D., Golden, R., Pedersen, C., Simon, J., \& Nemeroff, C. (1990). Subclinical hypothyroidism: A review of neuropsychiatric aspects. Int. J. Psych. Med., 20, 193-208.

Hamilton, M. (1967). Development of a rating scale for primary depressive illness. $B r$. J. Soc. Clin. Psychol., 6(4), 278-296.

Jaeshke, R., Guyatt, G., Gerstein, H., Patterson, C., Molloy, W., Cook, D., Harper, S., Griffith, L., \& Carbotte, R. (1996). Does treatment with L-thyroxine influence 
health status in middle-aged and older adults with subclinical hypothyroidism. Journal of General Internal Medicine, 11, 744-749.

Leentjens, A., \& Kappers, E. (1995). Persistent cognitive defects after corrected hypothyroidism. Psychopathology, 28, 235-237.

Mennemeier, M., Garner, R. D., \& Heilman, K. M. (1993). Memory, mood, and measurement in hypothyroidism. Journal of Clinical and Experimental Neuropsychology, 15(5), 822-831.

Monzani, E., Del-Guerra, P., Caraccio, N., Prunetic, C., Pucci, E., Luisi, M., \& Baschieri, L. (1993). Subclinical hypothyroidism: Neurobehavioral features and beneficial effect of L-thyroxine treatment. Clinical and Investigative Medicine, 71, 367-371.

Nebes, R., Butters, M., Mulsant, B., Pollock, B., Zmuda, M., Houck, P., \& Reynolds, C. (2000). Decreased working memory and processing speed mediate cognitive impairment in geriatric depression. Psychological Medicine, 30, 697-691.

Osterrieth, P. A. (1944). Le test de copie d'une figure complex: Contribution à l'étude de la perception et de la memoire. Archiv fur Psychologie, 30, 286-350.

Osterwil, D., Syndaulko, K., Cohen, S., Pettler-Jennings, P., Hershman, J., Cummings, J., Tourtelellotte, W., \& Solomon, D. (1992). Cognitive function in non-demented older adults with hypothyroidism. Journal of the American Geriatric Society, 40, 325-335.

Salthouse, T. (1991). Mediation of adult age differences in cognition by reductions in working memory and speed of processing. Psychological Science, 2, 179-183.

Sheehan, D. V., Lecrubier, Y., Sheehan, K. H., Amorim, P., Janavs, J., Weiller, E., Hergueta, T., Baker, R., \& Dunbar, G. C. (1998). The Mini-International Neuropsychiatric Interview (M.I.N.I): The development and validation of a structured diagnostic psychiatric interview for DSM-IV and ICD-10. J. Clin. Psychiatry, 59 (Suppl 20), 22-33, quiz 34-57.

Smith, J. W., Evan, A. T., Costall, B., \& Smythe, J. W. (2002). Thyroid hormones, brain function, and cognition: A brief review. Neuroscience Biobehavioral Review, 26(1), 45-60.

Wechsler, D. (1987). Wechsler Memory Scale—Revised. Manual. San Antonio: The Psychological Corporation.

Whybrow, P. C., \& Bauer, M. (2005). Behavioral and psychiatric aspects of hypothyroidism. In L. E. Braverman \& R. D. (Eds.), The thyroid. A fundamental and clinical text. 9th edn. (pp. 842-849). Philadelphia: Lippincott-Raven.

Whybrow, P. C., Prange, A. J., \& Treadway, C. R. (1969). Mental changes accompanying thyroid gland dysfunction. Archives of General Psychiatry, 20, 48-63. 